\title{
Composición Química del Aceite Esencial de Hojas de Orégano (Origanum vulgare)
}

\author{
Diofanor Acevedo, Mario Navarro y Luis Monroy \\ Universidad de Cartagena, Facultad de Ingeniería, Programa de ingeniería de Alimentos, Avda. el \\ Consulado, Calle 30 No. 48-152. Cartagena, Bolívar-Colombia (e-mail: diofanor3000@gmail.com)
}

* Autor a quien debe ser dirigida la correspondencia

Recibido Ene. 09, 2013; Aceptado Feb. 15, 2013; Versión final recibida Mar. 30, 2013

\section{Resumen}

Se ha analizado químicamente el orégano (Origanum vulgare) cultivado en Coloso, Departamento de Sucre en Colombia para determinar la variabilidad en la composición química causada por las condiciones climáticas de la región. Para ello se empleó cromatografía de gases acoplada con espectrometría de masas. Se identificó como compuesto mayoritario al timol con $67,51 \%$, seguido de p-cimeno, $y$-terpineno, cariofileno, oxido de cariofileno, trans- $\alpha$-bergamoteno, eugenol, y $\alpha$ - bergamoteno, con $11.66 \%, 5.51 \%$, $5.38 \%, 2.22 \%, 1.65 \%, 1.49 \%$, y $1.32 \%$ respectivamente. El Timol le otorga al orégano múltiples propiedades antioxidantes, microbiológicas y conservantes de alimentos, además de potenciales aplicaciones en perfumería y cosmética.

Palabras clave: origanum vulgare, aceite esencial, cromatografía de gases, espectrometría de masas

\section{Chemical Composition of the Essential Oil extracted from Oregano Leaves (Origanum vulgare)}

\begin{abstract}
The chemical composition of oregano (Origanum vulgare) cultivated in Coloso, Department of Sucre in Colombia has been determined with the objective of determining the variability in the chemical composition caused by climatic conditions of the zone. For this, gas chromatography coupled with mass spectrometry has been employed. The analysis allowed identifying Thymol as the major compound with $67.51 \%$, followed by $\mathrm{p}$-cymene, $\mathrm{\gamma}$-terpinene, caryophyllene, caryophyllene oxide, trans- $\alpha$-bergamoteno, eugenol and $\alpha$ bergamoteno, with $11.66 \%, 5.51 \%, 5.38 \%, 2.22 \%, 1.65 \%, 1.49 \%$ and $1.32 \%$ respectively. Thymol gives oregano several antioxidant, microbiologic and food preserving properties, besides potential applications in the perfume and cosmetic industry.
\end{abstract}

Keywords: origanum vulgare, essential oil, gas chromatography, mass spectrometry 


\section{INTRODUCCIÓN}

Los aceites esenciales (AE) son mezclas complejas de líquidos que presentan alta volatilidad, evaporándose al contacto con el aire (Bello, 1999). Son obtenidos a partir de diferentes partes de las plantas como flores, yemas, semillas, hojas, ramas, corteza, hierbas, madera, frutos y raíces (Burt, 2007). Químicamente están formados por terpenos, monoterpenos y sesquiterpenos (hidrocarburos, alcoholes, cetonas, que pueden ser acíclicos, monocíclicos, bicíclicos, tricíclicos), sustancias azufradas y nitrogenadas (Acevedo et al., 2007). Se consideran producto del metabolismo secundario de las plantas al igual que algunos alcaloides, flavonoides, taninos, y saponinas (Bandoni et al., 2009; Madsen \& Bertelsen, 1995). La composición química de los $\mathrm{AE}$ puede verse afectada por el medio ambiente, la procedencia de la planta y el método de extracción (Combariza et al., 1994). El valor económico de los AE y su aplicabilidad industrial están directamente relacionados con su composición química y con la actividad biológica (Stashenko et al., 2010). El creciente interés por el uso de extractos naturales antioxidantes que puedan sustituir los aditivos sintéticos en los alimentos, resulta una alternativa prometedora para la prevención y tratamiento de enfermedades producidas por compuestos sintéticos (Olivero-Verbel et al., 2010; Arcila-Lozano et al., 2004). De hecho muchos autores han reportado propiedades antimicrobianas, antifúngicas, antioxidantes y antiradicalarias de diferentes especias y aceites esenciales y en algunos casos, una aplicación directamente relacionada con los alimentos (Madsen \& Bertelsen, 1995). La extracción de estos se realiza por métodos convencionales como la destilación con arrastre de vapor y el uso de solventes orgánicos, aunque actualmente se utiliza la extracción supercrítica con dióxido de carbono como solvente (Simándi et al., 1998), de acuerdo con las propiedades de este gas (no tóxico, ni explosivo y fácil de remover), su uso facilita las diferentes operaciones de separación de los productos extraídos (Yépez et al., 2001). El análisis de quimiotipos se basa en la diferenciación de metabolitos secundarios y se aplica, no sólo a organismos vegetales, sino también a líquenes y algunos microorganismos (Arcila-Lozano et al., 2004). Por lo general para analizar un aceite esencial se utiliza la técnica de cromatografía de gases acoplada a espectrometría de masas (CG-EM), como técnica de identificación con base en los compuestos mayoritarios (Arcila-Lozano et al., 2004; Albado et al., 2001).

El orégano, (Origanum vulgare), pertenece a la familia Labiaceae, y es una planta herbácea vivaz muy aromática (Carhuapoma, 2006; Arcila-Lozano et al., 2004; D’Antuono et al., 2000). Sus hojas (tanto frescas como secas) se emplean como condimento en numerosas recetas culinarias por el excelente sabor que le confieren a las comidas (Albado et al., 2001). Se ha demostrado que el orégano contiene aceites esenciales, por lo que no solo es benéfico para la salud humana, sino que además puede sustituir los aditivos sintéticos de los alimentos (Arcila-Lozano et al., 2004). El AE de O. vulgare tiene actividad antiradicalaria y esta propiedad se le atribuye a los mono fenoles carvacrol y timol principales quimiotipos, cada una con enzimas específicas que dirigen su biosíntesis (D’Antuono et al., 2000; Deighton et al., 1993).

Sobre la composición química del orégano, y sus aceites esenciales se han identificado flavonoides como la apigenina y la luteolina, agliconas, alcoholes alifáticos, compuestos terpénicos y derivados del fenilpropano (Arcila-Lozano et al., 2004), también se han encontrado ácidos coumérico, ferúlico, caféico, rhidroxibenzóico y vainillínico (D’Antuono et al., 2000). La subespecie Oreganum vulgare ssp. Hirtum es la más estudiada, especialmente en relación a la composición y calidad de su aceite esencial, ya que este último tiene un importante valor comercial (Albado et al., 2001; Arcila-Lozano et al., 2004). En esta subespecie el rendimiento del aceite esencial en la hoja seca, varía entre $2 \%$ y 6\% (Deighton et al., 1993). Este porcentaje se ve afectado por la altitud del lugar de cultivo (Carhuapoma, 2006), y por la época de recolección, siendo este más bajo en el otoño (Arcila-Lozano et al., 2004). En el aceite del orégano que crece en forma silvestre se ha observado que un incremento en los porcentajes de timol provoca una disminución en el contenido de carvacrol (Albado et al., 2001). De igual manera, los hidrocarburos monoterpenoides, terpineno y r-cimeno están presentes de manera constante en los aceites esenciales del orégano, pero siempre en cantidades menores (Arcila-Lozano et al., 2004). Recientemente, Stashenko et al., (2010), caracterizaron tres grupos de orégano de monte presentes en Colombia en relación con la composición de los aceites esenciales: El Quimiotipo A que es rico en compuestos terpénicos (monoterpenos y sesquiterpenos que representan el 45 y $27 \%$, respectivamente); el Quimiotipo B, cuyo principal componente es el carvacrol (40\%) seguido de p-cimeno (13\%) y g-terpineno (11\%) y timol $(11 \%)$. Este quimiotipo es similar al encontrado en Brasil (Oliveira et al., 2004); el quimiotipo C posee altos niveles de timol (56\%, seguido de p-cimeno (9\%) y g- terpineno(5\%), este quimiotipo es similar a los reportados en Venezuela por Albado et al., (2001) y Arcila-Lozano et al., (2004).

Se han realizado diversos estudios sobre la bioactividad del orégano; es así como lo citan como antioxidante (Fasseas et al., 2007), antitripanosoma (Santoro et al., 2007), igualmente activo para un amplio grupo de bacterias patógenas (Albado et al., 2001). Asimismo, se ha aprovechado su doble ventaja en cuanto a las propiedades sensoriales y el tiempo de conservación del aceite esencial de orégano en pescado, prolongando el tiempo de vida útil del producto en comparación con otros métodos de conservación (Viuda-Martos et al., 2009). 
El objetivo del presente trabajo fue determinar la composición química del aceite esencial extraído de las hojas de orégano (O. vulgare) procedente del municipio de Coloso (Sucre-Colombia) por medio de la técnica de cromatografía de gases acoplada a espectrometría de masas.

\section{MATERIALES Y MÉTODOS}

\section{Obtención de la materia prima}

Las hojas de O. vulgare, fueron recogidas de parcelas artesanales ubicadas en el municipio de Coloso Departamento de Sucre Colombia. El material se recolecto de forma manual y se identificó en el herbario de Carsucrel. El modo de extracción que se utilizo fue hidrodestilación con trampa de Clevenger, el procedimiento consistió en picar finamente $1000 \mathrm{gr}$ de material fresco de hojas de $O$. vulgare, depositarlo en un recipiente de acero inoxidable, al que se le añadieron $2000 \mathrm{ml}$ de agua destilada, con un tiempo de extracción de 120-180 minutos.

\section{Caracterización química del aceite esencial de O. vulgare}

Los procedimientos para determinar la composición química del aceite esencial de orégano, se realizaron bajo la técnica de cromatografía de gases acoplada a espectrometría de masas (CG-EM).

Se necesitaron $50 \mu \mathrm{l}$ de aceite esencial para el análisis cromatográfico y se aforo a $450 \mu \mathrm{l}$ con diclorometano. La mezcla se realizó en un matraz aforado de $1.5 \mathrm{ml}$ y después se transfirió a un vial para cromatografía de gases de $2 \mathrm{ml}$. Este análisis se llevó a cabo en un Cromatógrafo de gases - Agilent Technologies 7890A, acoplado a un espectrómetro de masas Agilent Technologies 5975C equipado con un puerto de inyección Split/splitlles (relación 1:50), un inyector automático Agilent 7863 y un sistema de datos HP Chem Station.

Para la separación de las mezclas se utilizó una columna capilar DB-5MS( $J \& W$ Scientific, Folsom, EE.UU.) con fase estacionaria de $5 \%$ fenil-polimetilsiloxano $(60 \mathrm{~m} \times 0,25 \mathrm{~mm}$, D.I. $\times 0,25 \mu \mathrm{m}$, df). La temperatura del horno se programó desde $45^{\circ} \mathrm{C}(5 \mathrm{~min})$ hasta $150^{\circ} \mathrm{C}(2 \mathrm{~min})$ a $4^{\circ} \mathrm{C} / \mathrm{min}$, luego se incrementó hasta $250^{\circ} \mathrm{C}(5 \mathrm{~min})$ a $5^{\circ} \mathrm{C} / \mathrm{min}$, finalmente, la temperatura aumentó a $10^{\circ} \mathrm{C} / \mathrm{min}$, hasta alcanzar $275^{\circ} \mathrm{C}(15$ min). Las temperaturas de la cámara de ionización y de la línea de transferencia fueron de 230 y $285^{\circ} \mathrm{C}$, respectivamente. El gas de arrastre empleado fue helio (99.995\%). Los espectros de masas y corriente iónicas reconstruidas se obtuvieron por medio de barrido automático de frecuencia (full scan), a 4.75 scan s1 , en el rango de masas $\mathrm{m} / \mathrm{z} 30-450$.

Para el análisis por la columna polar se empleó un cromatógrafo de gases Agilent Technologies 6890 Network GC System, acoplado a un detector selectivo de masas Agilent Technologies 5975, equipado con un puerto de inyección split/splitless $\left(250^{\circ} \mathrm{C}\right.$, split 1:14) y un sistema de datos HP ChemStation D.02.00.275. El proceso de separación se llevó a cabo en una columna capilar DB-WAX ( $\mathrm{E} \& W$ Scientific, Folsom, CA, EE.UU.) con fase estacionaria entrecruzada e inmovilizada de polietilenglicol $(60 \mathrm{~m} \times 0,25 \mathrm{~mm}$, D.I. $\times 0,25$ $\mu \mathrm{m}, \mathrm{df})$. La temperatura del horno se programó desde $45^{\circ} \mathrm{C}(10 \mathrm{~min})$ hasta $220^{\circ} \mathrm{C}(30 \mathrm{~min})$ a $3^{\circ} \mathrm{C} / \mathrm{min}$. Las temperaturas de la cámara de ionización y de la línea de transferencia fueron de 230 y $250^{\circ} \mathrm{C}$, respectivamente. La identificación de los compuestos se realizó mediante la comparación de los tiempos de retención y de los patrones de fragmentación representados en los espectros de masas experimentales con los encontrados en las bases de datos o librerías (Data base INIST08.L, DatabaselW8.I).

\section{RESULTADOS}

Los resultados que se obtuvieron por GC-MS del aceite esencial de orégano tabla 1, revelan los principales componentes químicos, destacando el Timol (67.51\%) como mayoritario, seguido por p-Cimeno, $\mathrm{y}^{-}$ Terpineno, cariofileno, oxido de cariofileno, trans- $\alpha$-Bergamoteno, Eugenol, y a-Bergamoteno, con 11,66\%, $5,51 \%, 5,38 \%, 2,22 \%, 1,65 \%, 1,49 \%$, y $1,32 \%$ respectivamente, que representan más del $80 \%$ del total registrado (tabla1). Estos datos coinciden en gran medida con los informes de Amadio et al., (2011), donde el timol figura como componente mayoritario para la especie, sin embargo se presentan algunas diferencias cuantitativas en su composición, las que pueden ser debido a varios factores; en ese sentido Bandoni et al., (2009) expresaron que es casi imposible lograr dos aceites esenciales idénticos. Como resultado del grado de sensibilidad analítica con que se trabaje, siempre se podrán encontrar alguna diferencia entre dos partidas de un mismo aceite esencial, por la época de cosecha, el año, el método de extracción y las condiciones de almacenamiento. De igual forma, variables como las condiciones geobotánicas, tipo de suelo, época de recolección y edad de la planta (Stashenko et al., 2010; Bandoni et al., 2009; Albado et al., 2001), entre otros. 
Tabla 1: Componentes del aceite esencial de orégano (O. vulgare) identificados por GS-MS

\begin{tabular}{|c|c|c|c|}
\hline $\operatorname{Tr}(\min )$ & Compuesto & familia química & Área relativa (\%) \\
\hline 13,14 & p-cymene & Hidrocarburo monoterpénico & 11,66 \\
\hline 14,32 & y-Terpinene & Hidrocarburo monoterpénico & 5,51 \\
\hline 21,78 & Thymol & Compuesto oxigenado & 67,51 \\
\hline 23,21 & Eugenol & Compuesto oxigenado & 1,82 \\
\hline 24,89 & Caryophyllene & Sesquiterpeno bicíclico & 5,38 \\
\hline 25,26 & a-Bergamotene & Hidrocarburo sesquiterpénico & 1,32 \\
\hline 25,26 & trans-.alpha.-Bergamotene & Hidrocarburo sesquiterpénico & 1,65 \\
\hline 28,90 & Caryophyllene oxide & Terpenoide oxigenado & 2,22 \\
\hline
\end{tabular}

Los quimiotipos frecuentes en esta especie son quimiotipo Carvacrol y quimiotipo Timol. La importancia de esta planta se refleja en el alto valor agregado que adquiere su aceite esencial por sus componentes químicos, como el Timol $(67,51 \%)$.

El timol es un efectivo agente antimicrobiano que inhibe el crecimiento de microbios y bacterias por esta razón, el timol se utiliza en productos tales como enjuagues bucales que tienen como objetivo eliminar las bacterias en la boca y pastas de dientes, además de tener un sabor agradable .El timol se utiliza por vía oral en el tratamiento de la bronquitis, tosferina, dolor de garganta, diarrea, dispepsia, gastritis, desórdenes de la piel, desinfectante urinario y antihelmíntico. Tópicamente se utiliza en colutorios para prevenir las caries y para combatir la halitosis, para el tratamiento de la alopecia areata y también forma parte de muchos productos antiinflamatorios. Por sus propiedades antibacterianas y antifúngicas forma parte de gotas ópticas. El timol es también utilizado en cosmética y perfumería (Stashenko et al., 2010; Arcila Arcila-Lozano et al., 2004)

\section{CONCLUSIONES}

La composición química que presenta el aceite esencial de orégano cultivado en Coloso Sucre (Colombia) pertenece al quimiotipo Timol. Este compuesto permite dar gran valor agregado a esta planta, por su alto porcentaje en masa, que se convierte en posible fuente natural de este terpeno, y que le otorga múltiples propiedades antioxidantes, microbiológicas y conservantes de alimentos, así también como su uso en perfumería y cosmética dados las muchas aplicaciones como alternativas que se requieren en la industria.

\section{REFERENCIAS}

Acevedo A. y otros seis autores, Composición y capacidad antioxidante de especies aromáticas y medicinales con alto contenido de TIMOL Y CARVACROL, Scientia et Technica, 13(33), 125-128 (2007).

Albado, E., Saez, G. y Grabiel, S., Composición química y actividad antibacteriana del aceite esencial del Origanum vulgare (orégano). Revista Medica Herediana, 12(1), 17-19 (2001).

Amadio, C. y otros cinco autores, Aceite esencial de orégano: un potencial aditivo alimentario, Rev. FCA Uncuyo, 43(1), 237-245 (2011).

Arcila-Lozano, C. y otro tres autores, Orégano: Propiedades, composición y actividad biológica de sus componentes, ALAN, 54(1), 100-111 (2004).

Bandoni, A. y otros tres autores, ¿Son realmente útiles los aceites esenciales?, BLACPMA, 8(5), 317-22 (2009).

Bello, A., Estudios de aceites esenciales de especie Myrtaceae de la flora de Pinar del Río. Tesis de Maestría, Departamento de Ciencias Químicas, Universidad del rio, Rio, Brasil (1999).

Burt, S.A., Antibacterial activity of essential oils: potential applications in food, PhD thesis, Institute for Risk Assessment Sciences, Division of Veterinary Public Health, Utrecht University, Utrecht, Netherlands (2007).

Carhuapoma, M., Estudio de la composición química y actividad antioxidante del aceite esencial de Luma chequen (Molina) A. Gray "arrayán". Tesis de maestría, Facultad de farmacia y bioquímica, Universidad Nacional Mayor de San Marcos, Lima, Perú (2006). 
Combariza, M. y otros tres autores, Limonene concentration in lemon (Citrus volkameriana) peel oil as a function of ripeness, Journal of High Resolution Chromatography, 17(9), 643 - 646 (2005).

D’Antuono, L., Galletti, G. y Paola Bocchini, P., Variability of Essential Oil Content and Composition of Origanum vulgare L. Populations from a North Mediterranean Area (Liguria Region, Northern Italy), Annals of Botany, 86(3), 471-478 (2000).

Deighton, N. y otros tres autores, Identification by EPR spectroscopy of Carvacrol and Thymol as the major sources of free radicals in the oxidation of plant essential oils, J. Sci. Food Agric., 63(2), 221-225 (1993).

Fasseas, M. y otros cuatro autores, Antioxidant activity in meat treated with oregano and sage essential oils. Food Chemistry, 106(3), 1188-94 (2007).

Madsen, H. \& Bertelsen, G., Spices as antioxidants, Trends in food science \& technology, 6(8), 271-277 (1995).

Oliveira, D. y otros seis autores, Chemical and antimicrobial analyses of essencial oil of Lippia origanoides HBK, Food Chemistry, 101(1), 236-240 (2007).

Olivero-Verbel, J. y otros cuatro autores, Chemical composition and antioxidant activity of essential oils isolated from Colombian plants, Rev. bras. farmacogn., 20(4), 568-574 (2010).

Santoro, G. y otros cinco autores, Effect of oregano (Origanum vulgare L.) and thyme (Thymus vulgaris L.) essential oils on Trypanosoma cruzi (Protozoa: Kinetoplastida) growth and ultrastructure. Parasitol Res. 100(4), 783-90. (2007).

Simándi, B. y otros seis autores, Supercritical carbon dioxide extraction and fractionation of oregano oleoresin. Food Research International, 31(10), 723-728 (1998).

Stashenko, E. y otros seis autores, Lippia origanoides chemotype differentiation based on essential oil GCMS and principal component analysis, J. Sep. Sci., 33(1), 93-103 (2010).

Viuda-Martos, M. y otros tres autores, Effect of orange dietary fibre, oregano essential oil and packaging conditions on shelf-life of bologna sausages, Food Control. 21 (4), 436-43. (2009).

Yépez, B., Rivera, A. y López, S., Extracción de aceite esencial de limoncillo (Cymbopogon Citratus) con dióxido de carbono supercrítico, Congreso Colombiano de Ingeniería Química, 1-12, Santa Fe de Bogotá, Colombia 15 a 17 de agosto (2001). 
